\title{
Ovarian metastases from ileum cancer in a patient with germline EPCAM gene deletion successfully treated with surgical resection and CAPOX chemotherapy: a case report
}

Narushi Iwata', Ayumi Shikama1*, Wataru Takao ${ }^{1}$, Yoshihiko Hosokawa ${ }^{1}$, Hiroya Itagaki ${ }^{1}$, Nobutaka Tasaka ${ }^{1}$, Azusa Akiyama', Hiroyuki Ochi', Takeo Minaguchi', Miwa Arita², Emiko Noguchi' ${ }^{2}$, Toshikazu Moriwaki ${ }^{3}$ and Toyomi Satoh ${ }^{1}$

\begin{abstract}
Background: Despite recent findings that epithelial cell adhesion molecule (EPCAM) deletions can cause Lynch syndrome (LS), its clinical characteristics are still unknown. We present the first case of ileum cancer in a patient with germline EPCAM gene deletion, which was discovered during ovarian tumor surgery.

Case presentation: A 59-year-old woman presented with a history of colon cancer occurring at 38 and 55 years old. Five of her siblings had a history of colon cancer, and an elder sister had confirmed LS. As imaging examination revealed an ovarian tumor, and we performed hysterectomy and bilateral salpingo-oophorectomy. Careful observation during surgery revealed a cherry-sized tumor in the ileum, prompting partial ileal resection. Pathological examination showed the ovarian tumor to be a metastasis of ileum cancer. Genetic testing with blood-relative information using multiplex ligation-dependent probe amplification showed EPCAM exons 8 and 9 deletions, confirming LS. The patient received adjuvant chemotherapy with CAPOX (capecitabine and oxaliplatin) and has remained disease-free for 24 months.

Conclusions: We were fortunate to identify ileum cancer that would have been difficult to find preoperatively through careful observation during ovarian tumor surgery and successfully treated the patient by using surgical resection and CAPOX chemotherapy. When treating patients with hereditary cancer syndromes including LS, we should keep all associated cancers in mind.
\end{abstract}

Keywords: Lynch syndrome, EPCAM, lleum cancer, Ovarian metastases

\section{Background}

Lynch syndrome (LS) is caused by pathogenic germline variants in DNA mismatch repair (MMR) genes, $M L H 1$, MSH2, MSH6, and PMS2, and leads to a high risk of colorectal, endometrial, and several other extra-colonic

\footnotetext{
* Correspondence: ashikama@md.tsukuba.ac.jp

'Department of Obstetrics and Gynecology, Faculty of Medicine, University of Tsukuba, 1-1-1 Tennodai, Tsukuba, Ibaraki 305-8575, Japan

Full list of author information is available at the end of the article
}

cancers [1]. Recently, germline deletions in the epithelial cell adhesion molecule gene (EPCAM), which lies upstream of $M S H 2$, have been identified as a novel cause of LS. Deletion of the 3' region of EPCAM leads to subsequent epigenetic silencing of the $\mathrm{MSH} 2$ promoter region, resulting in MMR deficiency [2, 3].

EPCAM deletions account for about $1-3 \%$ of all pathogenic variants in LS [4]. The risk of colorectal cancer in individuals with EPCAM deletions is comparable

C C The Author(s). 2020 Open Access This article is licensed under a Creative Commons Attribution 4.0 International License, which permits use, sharing, adaptation, distribution and reproduction in any medium or format, as long as you give appropriate credit to the original author(s) and the source, provide a link to the Creative Commons licence, and indicate if changes were made. The images or other third party material in this article are included in the article's Creative Commons licence, unless indicated otherwise in a credit line to the material. If material is not included in the article's Creative Commons licence and your intended use is not permitted by statutory regulation or exceeds the permitted use, you will need to obtain permission directly from the copyright holder. To view a copy of this licence, visit http://creativecommons.org/licenses/by/4.0/ The Creative Commons Public Domain Dedication waiver (http://creativecommons.org/publicdomain/zero/1.0/) applies to the data made available in this article, unless otherwise stated in a credit line to the data. 
to those with MMR pathogenic variants in LS, whereas the cumulative risk of extra-colonic cancer is much lower compared to those with MMR pathogenic variants in LS [5]. However, there are few reports about LS patients with EPCAM deletions, and their clinical characteristics are still unknown.

Here, we present the first case of ileum cancer in a patient with germline EPCAM gene deletion, which was discovered during ovarian tumor surgery.

\section{Case presentation}

A 59-year-old woman visited her primary care physician for discomfort in the lower right abdomen. As no abnormalities were identified from gastroscopy or colonoscopy, her physician scheduled routine follow-up. Three months later, she again visited her physician complaining of hypertension. Serum carcinoembryonic antigen (CEA) level was elevated at $23 \mathrm{ng} / \mathrm{mL}$ (normal range, $0-5 \mathrm{ng} / \mathrm{mL}$ ), and computed tomography (CT) showed a $65-\mathrm{mm}$ tumor in the right ovary. She was referred to our hospital for further examination and treatment.

She developed transverse colon cancer at 38 years old and underwent transverse colectomy and lymph node dissection. At 55 years old, intramucosal colorectal cancer was diagnosed and endoscopically removed. The patient's family pedigree is shown in Fig. 1 . She had five siblings, four of whom (II- $1,-2,-4,-6)$ had a history of colorectal cancer and two of whom (II-1, -4) had a history of multiple colorectal cancers. EPCAM deletion was identified in an elder sister (II-2) and she underwent prophylactic hysterectomy and bilateral salpingooophorectomy in the United States.

Pelvic contrast-enhanced magnetic resonance imaging (MRI) revealed a tumor with solid components measuring $50 \times 78 \times 36 \mathrm{~mm}$ on the dorsal side of the uterus (Fig. 2). The interior of the tumor showed signal hyperintensity on $\mathrm{T} 2$-weighted imaging. The tumor showed lobular growth but had not infiltrated surrounding areas. Contrast-enhanced CT showed no distant metastases or lymph node metastases. Serum CEA level was elevated at $18 \mathrm{ng} / \mathrm{mL}$. Carbohydrate antigen 19-9 and carbohydrate antigen 125 levels were within normal ranges.

As the above findings suggested, the tumor could be either primary or secondary ovarian cancer. Therefore, we decided to perform surgery to allow for pathological examination. We performed total abdominal hysterectomy, bilateral salpingo-oophorectomy, and partial omentectomy. There was a fist-sized, lobular, cystic tumor in the right adnexa. The right ovarian tumor measured $8.5 \mathrm{~cm}$ in diameter, had a lobular interior, and was primarily mucinous with some solid portions (Fig. 3a). Ascites was absent, and the uterus and left adnexa appeared normal. During surgery, we found a cherry-sized tumor in the ileum, located on the $20-\mathrm{cm}$

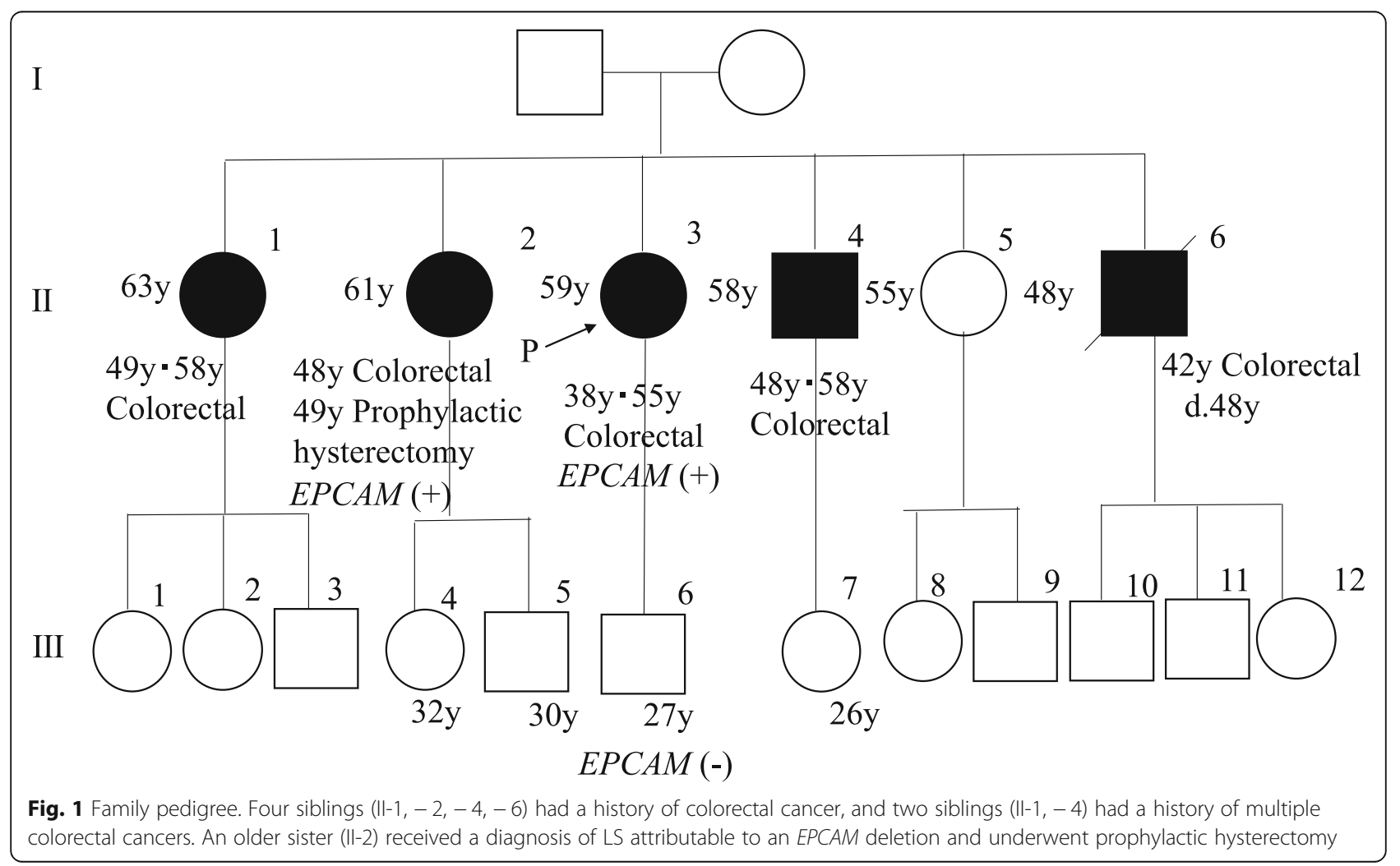




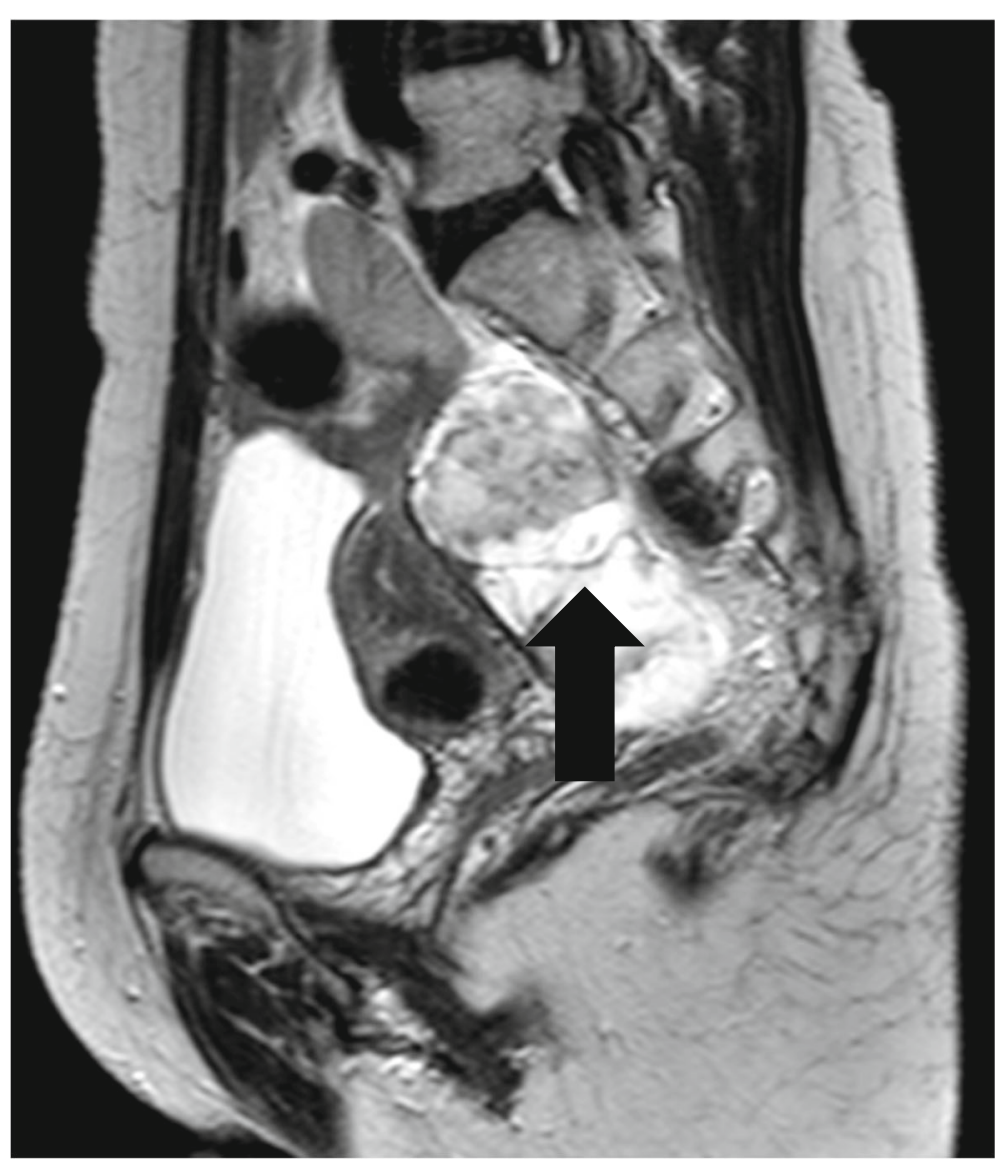

Fig. 2 Sagittal plane of the pelvic MRI. T2-weighted MRI of the pelvis reveals a tumor with solid components on the dorsal side of the uterus. The tumor interior shows signal hyperintensity

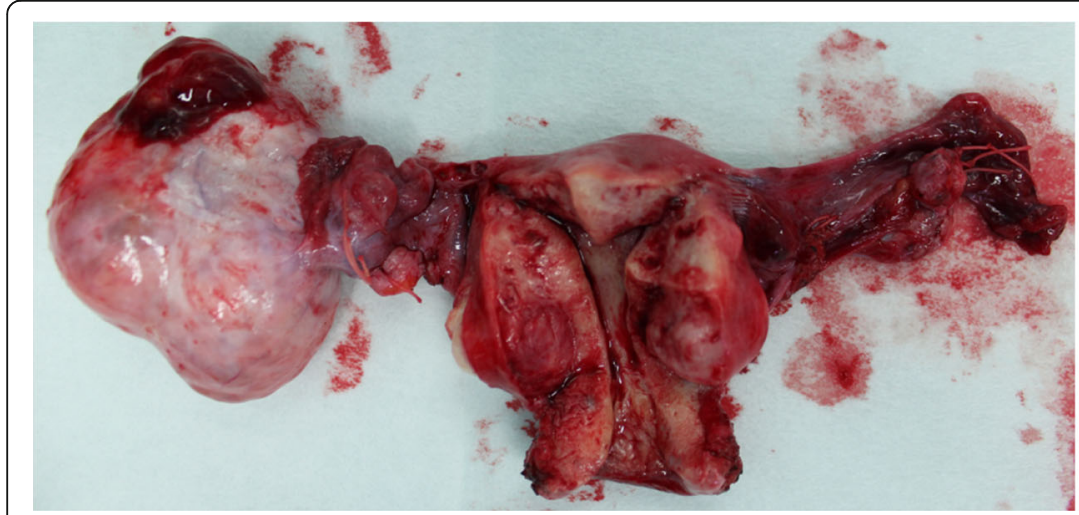

(a)

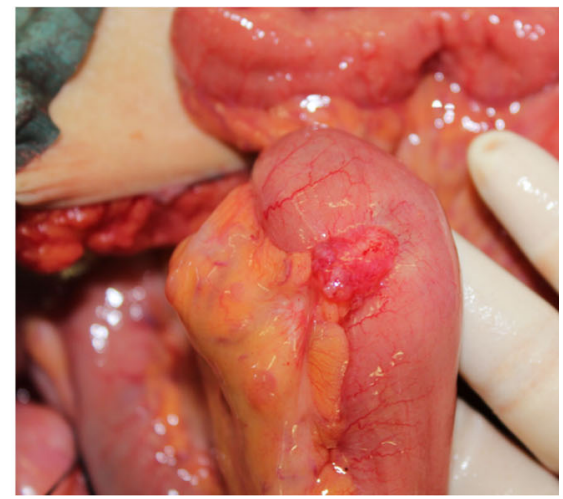

(b)

Fig. 3 Surgical findings. a The right ovarian tumor has a lobular interior, and is primarily mucinous with some solid portions. b The small bowel tumor shows papillary growth in the lumen 
mouth side from the ileocecum. Subsequent ileal resection was performed. The ileum tumor measured $2.5 \mathrm{~cm}$ in diameter and showed papillary growth in the lumen (Fig. 3b).

Histopathological examination revealed mucinous adenocarcinoma of the ileum. Atypical columnar cells growing in irregular/fused duct patterns were present in the ileum. Immunohistochemical staining of the tumor cells was negative for keratin 7 and keratin 20. The tumor had infiltrated to the subserosa and was partially exposed to the serosa (Fig. 4a, b). The ovarian tumor showed the same pathological features. Finally, the patient was diagnosed as having ileum cancer with ovarian metastasis (pT4NXM1, stage IV).

Furthermore, we performed immunohistochemical examination of MMR proteins. While MLH1 and PMS2 nuclear staining remained intact, $\mathrm{MSH} 2$ and MSH6 staining was decreased in both the ileum and ovarian tumor (Fig. 4c, d, e, f).

The patient recovered uneventfully and was discharged 10 days after surgery. We referred her to the Department of Gastroenterology of our hospital for adjuvant chemotherapy. She received chemotherapy comprising intravenous oxaliplatin $130 \mathrm{mg} / \mathrm{m}^{2}$ on day 1 and oral capecitabine $1000 \mathrm{mg} / \mathrm{m}^{2}$ twice daily from days 1 to 14 , every 3 weeks (CAPOX regimen). After four courses of CAPOX, the treatment regimen was switched to capecitabine monotherapy because of an oxaliplatin-related adverse event. She received two courses of capecitabine monotherapy, thereby completing initial treatment. Currently, she has remained disease-free for 24 months.

Because her medical and familial histories and immunohistochemical examination of MMR proteins suggested LS, we referred her for genetic counseling in the Department of Medical Genetics of our hospital. Based on genetic information from her older sister, genetic testing was performed with her consent. Multiplex ligation-dependent probe amplification assay showed deletions of EPCAM exons 8 and 9, upstream of $M S H 2$, confirming LS. Her son (III-6) also underwent genetic testing, but the results were negative.

\section{Discussion and conclusions}

This represents the first report of ileum cancer in a patient with germline EPCAM gene deletion, which is a novel cause of LS. The EPCAM gene encodes a type I transmembrane glycoprotein and is located $17 \mathrm{~kb}$ upstream of $M S H 2$ on chromosome 2. In most EPCAM carriers, germline deletions were found in regions including the polyadenylation site located in the last two exons (8 and 9), which lead to epigenetic silencing of MSH2 [6]. Because immunohistochemical staining for MMR proteins showed $M S H 2$ inactivation in the tumor tissue, ileum cancer in the present patient is very likely associated with LS.

Patients with LS have a 1-4\% lifetime cumulative incidence of small bowel cancer, about 100-times the lifetime risk in the general population [7]. Sporadic small bowel cancers generally occur in the duodenum, while LS-associated small bowel cancers frequently originate in other areas (43\% in duodenum, 37\% in jejunum, and $20 \%$ in ileum), showing a different distribution [8]. A study of 667 LS patients in the Netherlands found that 3 of 194 patients with EPCAM deletions (1.5\%) developed duodenal cancer. However, ileum cancer has not previously been reported in EPCAM carriers [9].

Few studies have documented the clinical features of EPCAM deletion with LS. Evaluating a population of 667 LS patients that included 194 patients with EPCAM deletion, Kempers and colleagues concluded that the risk of non-colorectal LS-associated cancers in EPCAM deletion carriers was lower than that in four MMR gene variants [9]]. Although the lifetime risk of colorectal cancer was similar at $75 \%$ in EPCAM deletion carriers and $70 \%$ in $M S H 2$ variants, the lifetime risk of endometrial cancer was $12 \%$ in EPCAM deletion carriers, but $51 \%$ in $M S H 2$ variants. They suggested that the risk of extra-colonic cancer may be associated with EPCAM deletions, depending on whether a deletion affects only the EPCAM gene or both EPCAM and $M S H 2$ genes. The present case revealed that ileum cancer was likely associated with LS, which indicates the need to consider small bowel cancer as LS-associated cancer in patients with EPCAM deletions.

Careful observation during ovarian tumor surgery allowed us to identify the ileum cancer that would have been difficult to find preoperatively in the patient. As clinical symptoms are frequently vague and non-specific, patients with ileum cancers are often diagnosed at advanced stages [10]. Furthermore, there are currently no effective methods to detect early-stage small bowel cancer in asymptomatic individuals. Video capsule endoscopy (VCE) and double-balloon endoscopy have recently emerged as alternatives to CT and MRI for identifying small bowel cancers. Haanstra and colleagues note that small bowel cancer can be overlooked in VCE [11]. In a prospective study evaluating the significance of VCE in diagnosing small bowel cancer in 200 patients with LS, 2 patients developed small bowel cancer. The disease was detected in one of the patients with VCE but was not found with VCE in the other, in whom stage II disease was diagnosed 7 months after negative VCE. VCE is not recommended for the surveillance of small bowel cancer in LS but is recommended for patients with symptoms suggestive of small bowel cancer [12].

The prognosis of small bowel cancer is poor, with 5year survival rates of $59.7 \%$ in the United States [13] and 

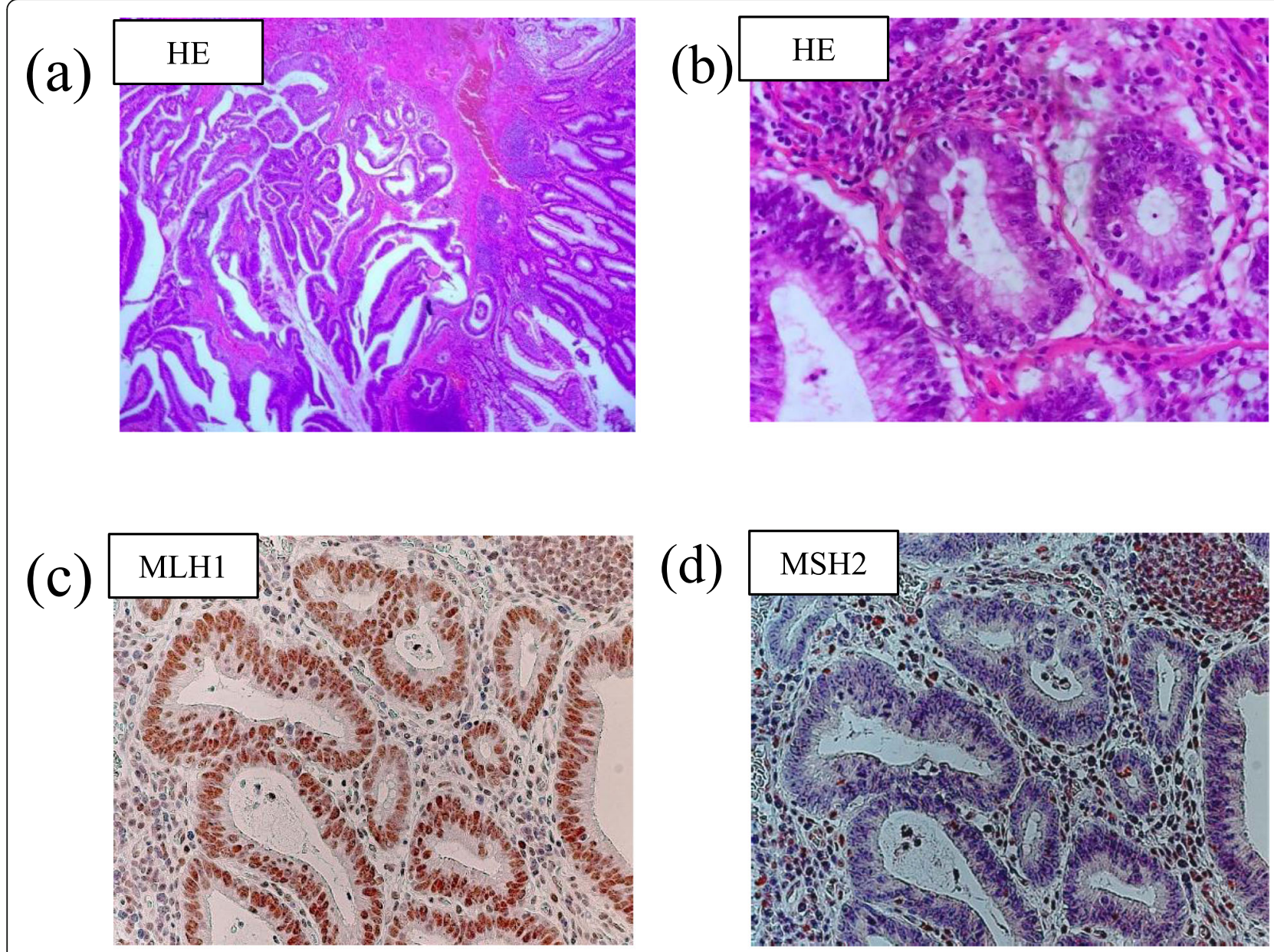

(e)

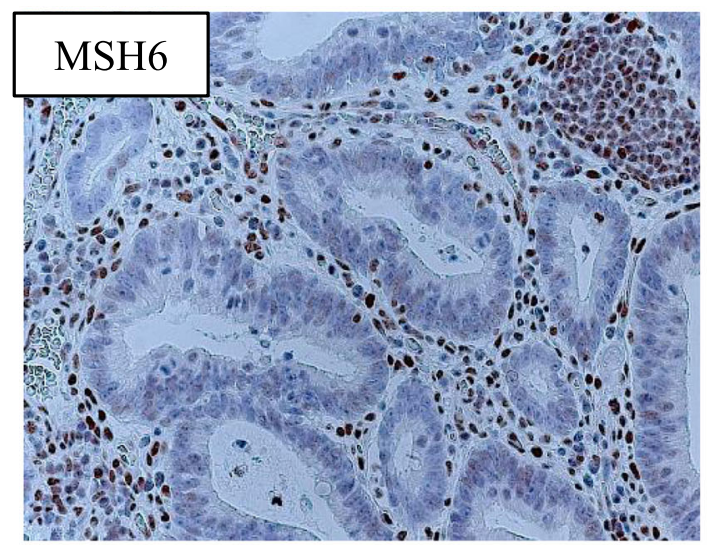

(f)

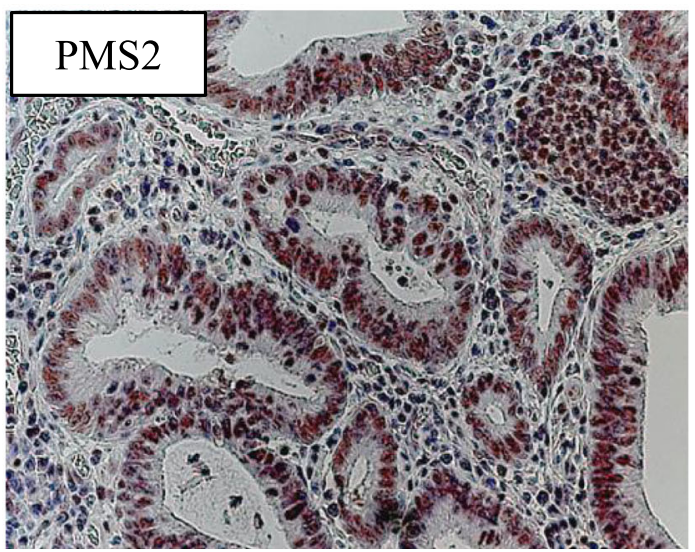

Fig. 4 Pathological examination of the small bowel tumor. Hematoxylin and eosin staining (a) 100x magnification and (b) 200x magnification. Atypical columnar cells growing in irregular/fused duct patterns are present. Immunohistochemical staining of MMR proteins (c) MLH1, (d) MSH2, (e) MSH6, (f) PMS2. The expression of MSH2 and MSH6 are decreased

48\% in Europe [14]. Given the rarity of small bowel cancer, no evidence-based standard treatment has been established. Surgical resection is the most common option for localized tumors. In a retrospective study performed in Japan, median overall survival in 10 patients with stage IV small bowel cancer who underwent surgical resection or local radiotherapy plus chemotherapy was 36.9 months [15], suggesting that a 
multidisciplinary approach could improve prognosis even in patients with distant metastases. Although the present patient was diagnosed at an advanced stage, complete resection was possible because the lesions were limited to the small bowel and ovary. She was subsequently treated using the CAPOX regimen because chemotherapy for small bowel cancer often includes fluoropyrimidine and platinum combination therapy according to gastric or colorectal cancer [16]. Surgery and adjuvant chemotherapy have kept the patient recurrence-free to date.

We reported here the first case of ileum cancer in a patient with germline EPCAM gene deletion, which can cause LS. Fortunately, we could identify ileum cancer that would have been difficult to find preoperatively through careful observation during ovarian tumor surgery and successfully treated the patient with surgical resection and CAPOX chemotherapy. Immunohistochemical staining for MMR proteins indicated the ileum cancer likely occurred as LS-associated cancer. When treating patients with hereditary cancer syndromes including LS, we should keep all associated cancers in mind.

\section{Abbreviations}

CAPOX: Capecitabine and oxaliplatin; CEA: Carcinoembryonic antigen;

$C T$ : Computed tomography; EPCAM: Epithelial cell adhesion molecule gene; LS: Lynch syndrome; MMR: DNA mismatch repair; MRI: Magnetic resonance imaging; VCE: Video capsule endoscopy

\section{Acknowledgments}

The authors gratefully acknowledge the medical staff who took care of this patient.

\section{Authors' contributions}

AS designed the case report and $\mathrm{NI}$ drafted the manuscript. TM1 revised the manuscript. $\mathrm{WT}, \mathrm{YH}$, and $\mathrm{HI}$ participate in the surgery of this patient. NT, AA, $\mathrm{HO}$ and TS discuss the diagnosis and treatment. TM2 took care of the patient with chemotherapy. MA and EN supported the diagnosis for Lynch syndrome. All authors have read and approved the final manuscript.

\section{Funding}

No specific funding was received for this study.

\section{Availability of data and materials}

The data showed in the report is not publicly available due to this containing information that could compromise the privacy of this patient and her family but is available from the corresponding author upon reasonable request.

\section{Ethics approval and consent to participate}

Ethical approval is not applicable for this manuscript. The authors obtained the patient's written consent to the major procedures for diagnosis and treatment.

\section{Consent for publication}

The authors obtained written informed consent from the patient to publish information on her disease and clinical course.

\section{Competing interests}

The authors declare that they have no competing interests.

\section{Author details}

'Department of Obstetrics and Gynecology, Faculty of Medicine, University of Tsukuba, 1-1-1 Tennodai, Tsukuba, Ibaraki 305-8575, Japan. ${ }^{2}$ Department of
Medical Genetics, Faculty of Medicine, University of Tsukuba, Tsukuba, Japan. ${ }^{3}$ Department of Gastroenterology, Faculty of Medicine, University of Tsukuba, Tsukuba, Japan.

Received: 21 October 2019 Accepted: 26 March 2020

Published online: 09 April 2020

\section{References}

1. Lynch HT, Boland CR, Gong G, Shaw TG, Lynch PM, Fodde R, Lynch JF, de la Chapelle A. Phenotypic and genotypic heterogeneity in the Lynch syndrome: diagnostic, surveillance and management implications. Eur J Hum Genetics. 2006;14(4):390-402.

2. Kovacs ME, Papp J, Szentirmay Z, Otto S, Olah E. Deletions removing the last exon of TACSTD1 constitute a distinct class of mutations predisposing to Lynch syndrome. Hum Mutat. 2009:30(2):197-203.

3. Ligtenberg MJ, Kuiper RP, Chan TL, Goossens M, Hebeda KM, Voorendt M, Lee TY, Bodmer D, Hoenselaar E, Hendriks-Cornelissen SJ, et al. Heritable somatic methylation and inactivation of MSH2 in families with Lynch syndrome due to deletion of the $3^{\prime}$ exons of TACSTD1. Nat Genet. 2009; $41(1): 112-7$

4. Kuiper RP, Vissers LE, Venkatachalam R, Bodmer D, Hoenselaar E, Goossens M, Haufe A, Kamping E, Niessen RC, Hogervorst FB, et al. Recurrence and variability of germline EPCAM deletions in Lynch syndrome. Hum Mutat. 2011;32(4):407-14.

5. Ligtenberg MJ, Kuiper RP, van Kessel AG, Hoogerbrugge N. EPCAM deletion carriers constitute a unique subgroup of Lynch syndrome patients. Familial Cancer. 2013;12(2):169-74.

6. Tutlewska K, Lubinski J, Kurzawski G. Germline deletions in the EPCAM gene as a cause of Lynch syndrome - literature review. Hereditary Cancer Clin Pract. 2013;11(1):9.

7. Sun KK, Liu G, Shen X, Wu X. Small bowel adenocarcinoma in Lynch syndrome: a case report. Oncol Lett. 2016;12(2):1602-4.

8. Sanchez-Mete L, Stigliano V. Update on small bowel surveillance in hereditary colorectal cancer syndromes. Tumori. 2018;105(1):12-21.

9. Kempers MJ, Kuiper RP, Ockeloen CW, Chappuis PO, Hutter P, Rahner N, Schackert HK, Steinke V, Holinski-Feder E, Morak M, et al. Risk of colorectal and endometrial cancers in EPCAM deletion-positive Lynch syndrome: a cohort study. Lancet Oncol. 2011;12(1):49-55.

10. Azizi AH, Inam ZS, Farrell TJ. Patient with Lynch syndrome with subsequent development of small bowel adenocarcinoma. BMJ Case Rep. 2018;2018. https://doi.org/10.1136/bcr-2018-225003.

11. Haanstra JF, Al-Toma A, Dekker E, Vanhoutvin SA, Nagengast FM, MathusVliegen EM, van Leerdam ME, de Vos tot Nederveen Cappel WH, Sanduleanu $\mathrm{S}$, Veenendaal RA, et al. Prevalence of small-bowel neoplasia in Lynch syndrome assessed by video capsule endoscopy. Gut. 2015;64(10):1578-83.

12. Vasen HF, Blanco I, Aktan-Collan K, Gopie JP, Alonso A, Aretz S, Bernstein I, Bertario L, Burn J, Capella G, et al. Revised guidelines for the clinical management of Lynch syndrome (HNPCC): recommendations by a group of European experts. Gut. 2013;62(6):812-23.

13. Qubaiah O, Devesa SS, Platz CE, Huycke MM, Dores GM. Small intestinal cancer: a population-based study of incidence and survival patterns in the United States, 1992 to 2006. Cancer Epidemiol Biomarkers Prev. 2010;19(8):1908-18.

14. Anderson LA, Tavilla A, Brenner H, Luttmann S, Navarro C, Gavin AT, Holleczek B, Johnston BT, Cook MB, Bannon F, et al. Survival for oesophageal, stomach and small intestine cancers in Europe 1999-2007: Results from EUROCARE-5. Eur J Cancer (Oxford, England : 1990). 2015;51(15):2144-57.

15. Sakae H, Kanzaki H, Nasu J, Akimoto Y, Matsueda K, Yoshioka M, Nakagawa M, Hori S, Inoue M, Inaba T, et al. The characteristics and outcomes of small bowel adenocarcinoma: a multicentre retrospective observational study. Br J Cancer. 2017;117(11):1607-13.

16. Locher C, Batumona B, Afchain P, Carrere N, Samalin E, Cellier C, Aparicio T, Becouarn Y, Bedenne L, Michel P, et al. Small bowel adenocarcinoma: French intergroup clinical practice guidelines for diagnosis, treatments and follow-up (SNFGE, FFCD, GERCOR, UNICANCER, SFCD, SFED, SFRO). Digest Liver Dis. 2018;50(1):15-9.

\section{Publisher's Note}

Springer Nature remains neutral with regard to jurisdictional claims in published maps and institutional affiliations. 